\section{The Transmissible Nature of Hepatitis B and EB Viruses}

\section{To the Editor:}

The general medical epidemiologic and gastrointestinal literature is well supplied with citations documenting the transmissible nature of hepatitis $B$ virus and $\mathrm{EB}$ virus in respiratory secretions and saliva. The assumption is that the communicable nature of these viruses is based on the contention that infected saliva is transmitted on the tip of the "passed around" cigarette. This would further be supported by the fact that such activities are commonly undertaken in small groups and repeated exposure of a positive patient to unknown subjects would increase the risk of infectivity. This observation has been made on a historical and clinical basis in my practice.

Jack Goldstein, MD

Department of Gastroenterology Rancocas Valley Division Zurbrugg Memorial Hospital Willingboro, New Jersey

\section{Double Clean Operations in the Same Operating Room}

\section{To the Editor:}

Surgeons who practice in both of our hospitals have requested that our Infection Control Committees approve the performance of double clean operations in the same operating room when feasible and when acceptable by the patient.

We have checked with other hospitals and licensing agencies and found that this is not a common practice and no one seems to be aware of any literature or data concerning the matter.

Our hospitals are physically con- nected and are affiliated with a medical school. We are concerned about implementing this practice from an infection control point of view and request your professional opinion regarding this concept.

Linda D. Howell, BS/MT (ASCP) Infection Control Coordinator Norfolk General Hospital Norfolk, Virginia

Donna M. Sandifer, RN, CIC Nurse Epidemiologist Children's Hospital of The King's Daughters Norfolk, Virginia

\section{Dr. Laufman was asked to respond.}

There is no literature on the virtues or faults of performance of two clean children's operations in the same operating room. I know of no regulations or guidelines limiting the number of operating tables in one room.

You may or may not know that in some countries (Russia, for example, by my personal observation) more than two clean adult operations are being done in large operating theatres, even to the extent of sharing the same clean back table between two operating tables as backup supply for instruments.

In an Evacuation Hospital in World War II (my war) we operated on battle casualties in a large surgical tent, using as many as five side-by-side operating tables in the same large space. Of course, we left the wounds open for delayed secondary closure except for belly and chest wounds. This procedure was done by Army directive to guard against wound infection. One can hardly consider this situation to be parallel to your question about doing two clean children's operations in the same room, except to point out that it can be done even when the operations are not really clean. One can always find ways of safeguarding against infection such as leaving the wounds open.
In Pretoria, South Africa, the cleanest operations (joint replacements) are being done in cubicles separated only by plastic sheet walls in a large space. The anesthetists sit outside the plastic encasement while the surgeons and patient are inside.

My main concerns about doing more than one operation in the same room at the same time would be that: 1) the cases are truly clean, ie, herniorrhaphies, etc., and not mixing with potentially contaminated cases, ie, acute appendicitis; 2) each operating table should have its own back instrument table; 3) the room be large enough to accommodate both teams and circulating help without danger of contact, or have a plastic sheet divider separating the two operating areas.

Harold Laufman, MD, PhD, FACS Surgical Consultant New York, New York

\section{Corrections in Readers' Forum}

\section{To the Editor:}

There are two errors in "Readers' Forum: A Perspective on Controlling Vaccine-Preventable Diseases Among Children in Liberia" published in the November 1984 issue of Infection Control. Both errors are found on page 539 , in the second column, second paragraph, third sentence. You will note that this sentence is incomplete and duplicated. Also it contains the word "native," a term which I did not use. Please note that the sentence should read:

"In place of these, emphasis must be directed toward developing alternative areaspecific strategies to deliver immunizations with existing resources."

R. Mark Weeks, MPH Memphis, Tennessee 